\title{
Imaging Belgian Chocolate by Field Ion Microscopy
}

\author{
Luc Jacobs $^{1}$, Thierry Visart de Bocarmé ${ }^{1}$ and Cédric Barroo ${ }^{1 *}$ \\ ${ }^{1}$ Chemical Physics of Materials and Catalysis, Université libre de Bruxelles, CP243, 1050 Brussels, \\ Belgium \\ * Corresponding author: cbarroo@ulb.ac.be
}

The structure of commercial chocolate bars consists of a suspension of milk, sucrose and solid cocoa particles embedded in a continuous semi-solid 'fat phase', their ratio depending on the type of chocolate. The resulting cocoa butter can present six different crystallographic structures with their own densities and melting points, affecting the chocolate properties, such as the fat bloom formation, and induce different sensory perceptions. A recent study proved that it was possible to image dark chocolate samples by atom probe tomography and suggested that further experiments were required to explore the possibility of observing crystallography on chocolate samples [1]. In this work, we used field emission techniques to observe such crystallographic structure at the nanoscale. Field emission techniques include field emission microscopy (FEM) and field ion microscopy (FIM). The extremity of very sharp tipsamples can be imaged using the emission of electrons (FEM-mode) or the ionization of an imaging gas (FIM-mode). With a magnification of $10^{6}$ and a typical resolution in FIM of $0.2 \mathrm{~nm}$, these methods are particularly suited to image the atomic structure of metallic surfaces and their changes due to adsorption or reaction processes, but also to analyse the arrangement of thin films covering the tip-sample. In the golden ages of FIM, different organic molecules have been used as imaging gases [2-3], or organic- and bio-molecules adsorbed on clean tips (polymer layers, DNA...) have been imaged and can produce structured images in low field strength imaging [4-6]. Chocolate is thus a perfect model system to image the crystallographic structure of food samples.

Tungsten and rhodium tips were electrochemically etched from high purity wires using the microelectropolishing method in a $\mathrm{NaOH}$ solution $\left(2 \mathrm{M}, \mathrm{V}_{\mathrm{DC}}=3 \mathrm{~V}\right)$ for $\mathrm{W}$ tips, and in a molten salt mixture of $\mathrm{NaCl} / \mathrm{NaNO}_{3}(1: 4 \mathrm{w} / \mathrm{w})\left(520^{\circ} \mathrm{C}, \mathrm{V}_{\mathrm{DC}}=2.1 \mathrm{~V}\right)$ for Rh tips. The tips were characterized by both FEM and FIM prior to the deposition of the material of interest. A specific Belgian chocolate was selected, identic to the samples used for atom probe tomography analysis in [1]. The deposition of a thin layer of chocolate was performed using two different techniques (Figure 1.a-b). The first one uses the microelectropolishing setup: solid chocolate is deposited within the Pt ring, heated with a blow torch, and a micro-manipulator allows to dip the tip in the melted chocolate. The second method consists of the "bain-marie" technique and allows for a better control in the temperature of the chocolate materials, therefore avoiding phase separation. The tip is then manually dipped into the melted chocolate while avoiding direct contact between the apex part of the tip and the chocolate, avoiding the bending of the tip due to the surface tension of melted chocolate.

At low imaging field and low temperature $(T=65 \mathrm{~K})$, we can observe the presence of an increasing number of bright spots that remain and evolve towards a structured pattern. However, no specific crystallography can be discerned. By further increasing the imaging field, the removal of the structured layer can be observed via a sudden desorption revealing the underlying metallic sample. The fast desorption 'all at once' is characteristic of the removal of refractory layers. Therefore, the structured pattern present before desorption corresponds to a thin chocolate-layer. With the presence of chocolate on the shank of the tip, as can be observed by optical microscopy (Figure 1), it is possible to redeposit a 
layer of chocolate at the very apex part of the tip. To do so, the sample was heated at temperatures up to $310 \mathrm{~K}$ in absence of electric field, but also while imaging in FEM and FIM. In FEM mode, at constant imaging field, the diffusion of chocolate translates into the disappearance of the FEM pattern. A higher field is necessary to image the chocolate-covered sample and a new FEM pattern emerges (Figure 1.cd). In FIM mode, a more brutal behavior is observed, and when the temperature is decreased, a new FIM pattern is observed, corresponding to a FIM-chocolate pattern. This layer can be desorbed by gentle field evaporation or by desorption until the clean metallic sample is recovered (Figure 2.a-d).

To conclude, FIM allows to observe the behavior of complex organic molecules: field evaporation, field induced desorption, and re-adsorption by diffusion. Both FEM and FIM patterns exhibit patterns which are specific of the thin film. More importantly, this work confirms the possibility of observing crystallography of chocolate samples [1], proving the importance of combining FIM imaging with atom probe tomography experiments to get a better understanding of the systems studied [7].

References:

[1] C. Barroo, A. J. Akey and D. C. Bell, Microsc. Microanal. 23(S1) (2017) 708-709.

[2] B. Schulze et al., Appl. Surf. Sci. 87-88 (1995) 140-145.

[3] Y. Neo et al., Jpn. J. Appl. Phys. 51 (2012) 115601.

[4] A. Fischer et al., J. Vac. Sci. Technol. B 36 (2018) 051201.

[5] K. Kakuta et al., Conference Record - IAS Annual Meeting (2008) 4658900.

[6] E.S. Machlin et al., J. Microsc. 104 (1975) 127-168.

[7] L. J. and C.B. thank the Fonds de la Recherche Scientifique (F.R.S.-FNRS) for financial support

(PhD grant from FRIA and Postdoctoral fellowship from FNRS).
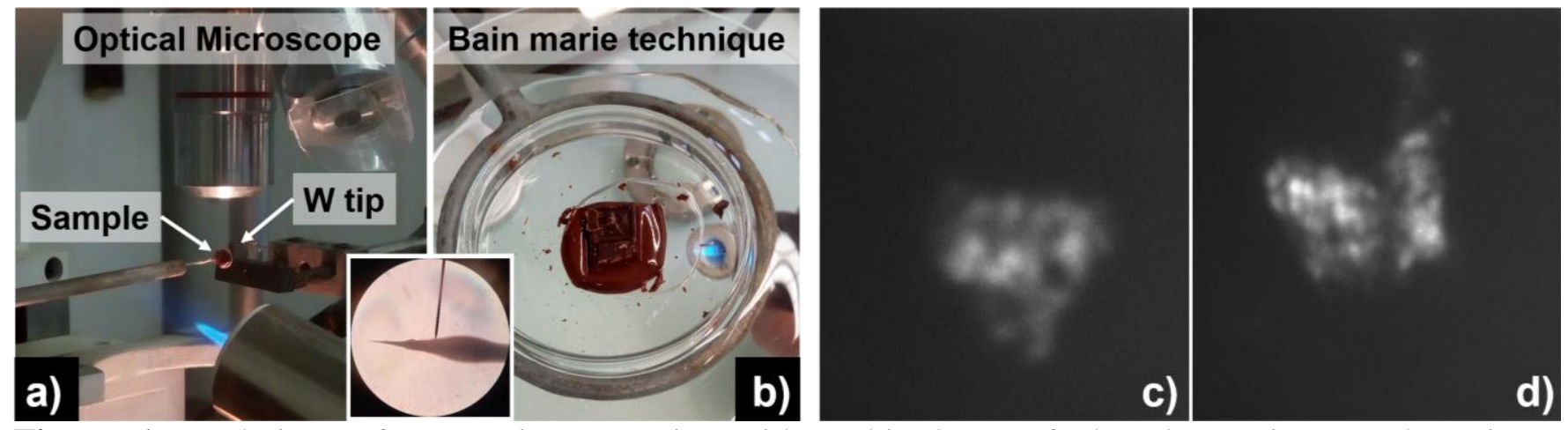

Figure 1. Techniques for covering FIM-tips with a thin layer of chocolate using: a. the microelectropolishing setup and b. the 'bain-marie' technique; FEM patterns of a Rh sample c. in absence $(1,23 \mathrm{kV})$ and $\mathbf{d}$. presence $(2,24 \mathrm{kV})$ of a chocolate layer highlighting the different symmetry.
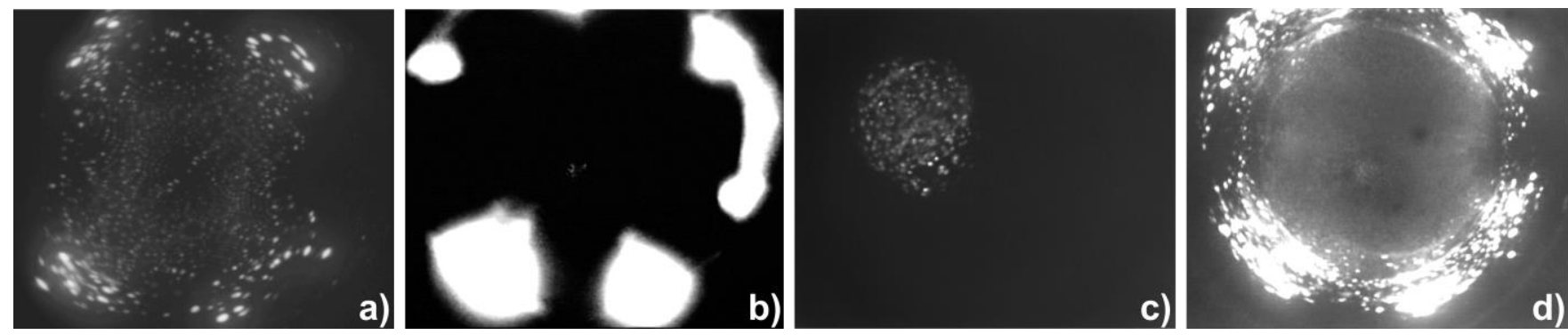

Figure 2. FIM imaging a. clean $\mathrm{W}$ tip $(\mathrm{T}=65 \mathrm{~K})$; b. during heating to recover the apex with chocolate $(\mathrm{T} \approx 300 \mathrm{~K})$; c. chocolate-covered $\mathrm{W}$ tip; d. clean $\mathrm{W}$ tip right after chocolate desorption. 